\title{
Avaliação das reações adversas ao uso de contrastes em exames de diagnóstico por imagem
}

\author{
Evaluation of adverse reactions to contrast use \\ in imaging diagnostic tests
}

Adriane Pozzobon, ${ }^{1}$ Fernanda Rocha da Trindade ${ }^{1}$

'Universidade do Vale do Taquari (Univates), Lajeado, RS, Brasil.

Recebido em: 07/09/2017 / Aceito em: 01/10/2017 / Publicado em: 01/10/2017 pozzobon@univates.br

\section{RESUMO}

Para maior precisão e diferenciação de estruturas anatômicas, os contrastes são amplamente usados em exames de diagnóstico por imagem, contudo seu uso indiscriminado pode favorecer o aparecimento de reações adversas no organismo. Objetivo: realizar uma revisão sistemática sobre os efeitos adversos relacionados ao uso de contrastes utilizados em exames de tomografia computadorizada e ressonância magnética. Método: foi realizada uma busca nas bases de dados PubMed e SciELO. Os termos descritores empregados foram: efeitos adversos (adverse effects), meio de contraste iodado (iodinated contrast media), meio de contraste (medium contras) e gadolínio (gadolinium) cruzados aleatoriamente. Foram incluídos artigos originais disponíveis na íntegra, publicados entre 2005 e 2016. Resultados: foram selecionados 21 artigos em que, verificou-se maior prevalência de efeitos adversos associados ao uso de contrastes, contudo, tanto nos contrastes iodados, quanto à base de gadolínio, a maioria das reações adversas foi classificada como leve, incluindo principalmente manifestações cutâneas. Considerações finais: apesar da maioria dos efeitos adversos estarem associados aos contrastes iodados, os mecanismos envolvidos no desenvolvimento dos efeitos adversos permanecem desconhecidos.

Palavras-chave: Meios de contraste; Gadolínio; Ressonância Magnética; Tomografia Computadorizada.

\section{ABSTRACT}

For greater precision and differentiation of anatomical structures, the contrasts are widely used in diagnostic imaging tests, but their indiscriminate use may favor the emergence of adverse reactions in patients. Objective: to perform a systematic review on the adverse effects related to the use of contrasts used in computed tomography and magnetic resonance tests. Method: we searched the PubMed and SciELO databases. The terms descriptors employed were: adverse effects, iodinated contrast media, contrast medium and gadolinium (randomly crossed). Full original articles published between 2005 and 2016 were included. Results: twenty-two articles were selected in which there was a higher prevalence of adverse effects associated with the use of contrasts, however, in both iodinated and gadolinium-based contrasts, most adverse reactions were classified as mild, including mainly cutaneous manifestations. Closing remarks: although the majority of adverse effects are associated with iodinated contrast, the mechanisms involved in the development of adverse effects remain unknown.

Keywords: Contrast media; Gadolinium; Magnetic resonance; Computed tomography. 


\section{INTRODUÇÃO}

Os Raios X foram descobertos em oito de novembro de 1895 por Wilhelm Conrad Roentgen (1845-1923), enquanto procurava detectar a radiação eletromagnética de alta frequência, prevista por Heinrich Hertz (1857-1894). Logo após sua descoberta, a primeira aplicação clínica foi na visualização das estruturas ósseas. A Tomografia surgiu na década de 70, pelo engenheiro Godfrey Housnfield e realizava apenas imagens do cérebro, sendo posteriormente aprimorada para a visualização de outros tecidos. ${ }^{1}$ O desenvolvimento de novos equipamentos de imagem vem contribuindo com diagnóstico mais preciso, melhor qualidade e aumento da expectativa de vida. Estima-se que no Brasil, existam de cerca de 15.217 equipamentos de imagem na rede pública e 37.610 na rede privada. ${ }^{2} \mathrm{Um}$ estudo mostrou que apesar das diferenças econômicas entre as regiões brasileiras, a oferta de equipamentos de imagem vem aumentando nos últimos anos. Só no estado de São Paulo, a média de realização de exames por imagem pelo Sistema Único de Saúde é estimada em três exames por ano para cada habitante..$^{3,4}$

A Tomografia Computadoriza (TC) consiste em aplicar diversas medidas de transmissão dos fótons de raios $X$, em múltiplos ângulos e, a partir desses valores calculam-se os coeficientes de absorção pelos diversos tecidos. ${ }^{5}$ A Ressonância Magnética (RM) é a propriedade física exibida por núcleos de determinados elementos que, quando submetidos a um campo magnético externo forte e excitados por ondas de Radiofrequência (RF), o qual pode ser captado por uma antena e transformado em imagem. Um dos grandes avanços da RM, é que ela usa radiação não-ionizante, não sendo lesiva para os tecidos biológicos, além de apresentar grande resolução para as partes moles, e com boa diferenciação entre as estruturas anatômicas. Ainda, o mesmo equipamento proporciona imagens anatômicas e também imagens funcionais. ${ }^{6}$

Após a descoberta dos Raios-X, constatou-se que estruturas com opacidades semelhantes não eram diferenciadas, tais como, os vasos sanguíneos. Desta forma, surgiu a necessidade de criação de métodos artificiais para aumentar o contraste entre estas estruturas. $\mathrm{Na}$ década de 50, Wallingford sintetizou o ácido benzoico tri-iodado, com base no qual foram sintetizados, produtos de contraste hiperosmolares, com uma osmolalidade cinco a oito vezes superior à do plasma e que se tornaram referência nos anos seguintes. ${ }^{7}$ Contudo os contrastes iodados utilizados apresentam muitos efeitos adversos, sendo estes resultantes da sua dissociação iônica, após injeção intravascular, com a criação consequente de cargas elétricas e hiperosmolaridade plasmática. ${ }^{8}$

Nos exames de RM, os principais contrastes empregados são à base de quelantes do íon paramagnético gadolínio $(\mathrm{Gd}) .^{9} \mathrm{Em}$ comparação ao contraste iodado, o Gd possui maior sensibilidade, além do fato que o volume médio de contraste administrado por via intravenosa nos exames de RM é de cinco a 15 vezes menor do que o utilizado com os contrastes iodados na TC. Este é um dos motivos pelo qual o uso do Gd é mais seguro do que os demais contrastes iodados..$^{10}$ Diante do exposto, o presente trabalho teve como objetivo verificar através de uma revisão sistemática, artigos que documentam a prevalência e os efeitos adversos relacionados ao uso de meios de contraste usados em TC e RM.

\section{MÉTODO}

A presente pesquisa é um estudo de caráter descritivo, transversal, quali-quantitativo. Foram coletados artigos nas bases de dados PubMed e Scientific Eletronic Library on-line (SciELO). Os termos descritores empregados foram: efeitos adversos (adverse effects), meio de contraste iodado (iodinated contrast media), gadolínio (gadolinium) e meio de contraste (medium contrast) cruzados aleatoriamente. Foram incluídos estudos de revisão e artigos originais disponíveis na íntegra, publicados entre 2005 e 2016. A revisão foi realizada em abril de 2016. A revisão sistemática do presente estudo foi norteada pela seguinte questão de pesquisa: Qual a prevalência e o impacto das reações adversas aos meios de contraste usados em TC e RM? Foram eleitos os seguintes critérios de inclusão: (a) estudos com seres humanos; (b) texto em língua inglesa ou em português; (c) artigos publicados na íntegra com acesso livre; (d) publicação em um periódico revisado por pares (peer-reviewd). Foram excluídos os artigos que não se enquadraram nos critérios de inclusão.

\section{RESULTADOS}

A busca inicial no PubMed com as palavras: iodinate medium contrast adverse effects localizou 136 artigos entre 2005 e 2016. Para a análise dos efeitos dos contrastes iodados foram selecionados 12 artigos originais disponíveis que preenchiam os critérios de inclusão. Foram excluídos os estudos que não preenchiam os critérios de inclusão. No SciELO foram localizados três artigos, e apenas um foi incluído no presente estudo por se referir a efeitos adversos de contraste iodado (Figura 1).

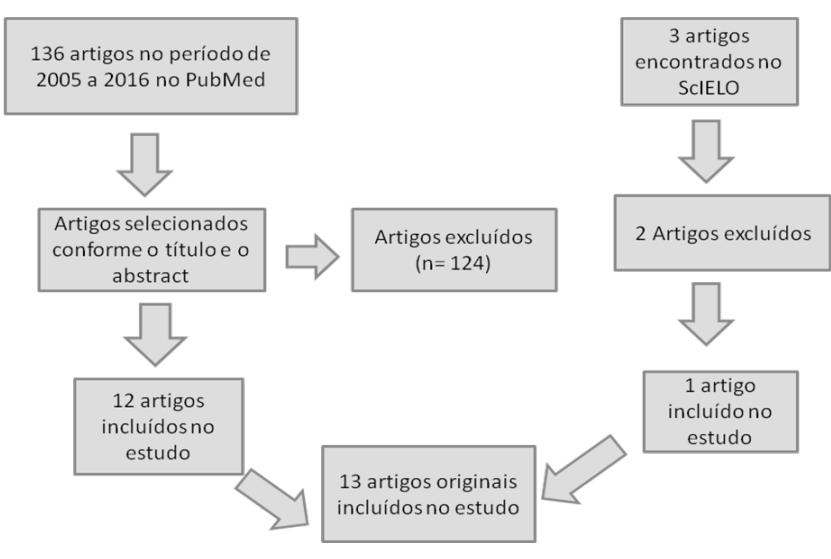

Figura 1 - Resultados da pesquisa indicando os trabaIhos selecionados com relação ao tema efeitos adversos ao uso de contraste iodado.

Com relação aos efeitos adversos do gadolínio, empregando as palavras: gadolinium medium contrast adverse effects foram encontrados 48 artigos entre 2005 e 2016 no PubMed, sendo incluídos na presente 
pesquisa, oito artigos e os demais excluídos por não preencher os critérios de inclusão. Não foram encontrados estudos sobre este tema na base de dados SciELO (Figura 2).

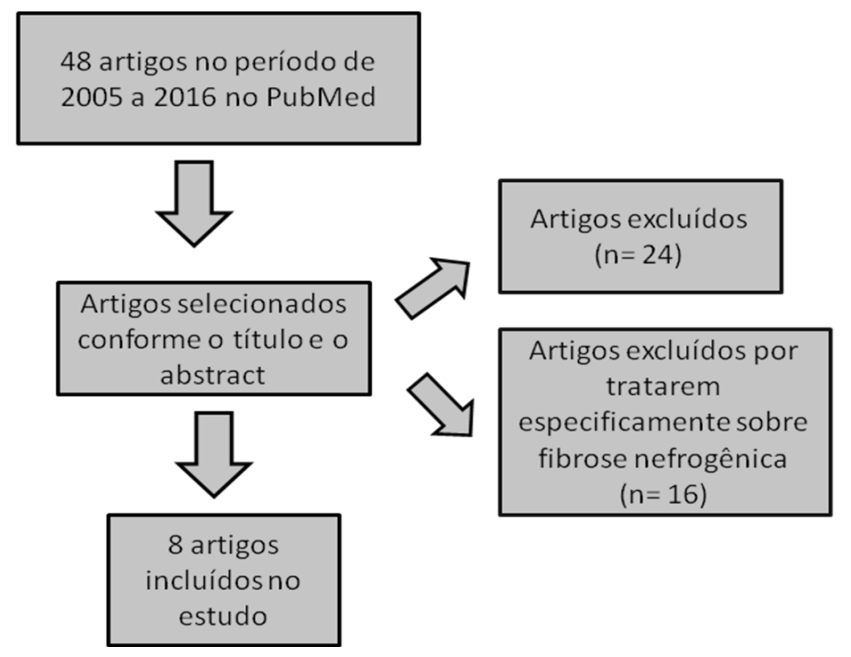

Figura 2 - Resultados da pesquisa com relação aos efeitos adversos do gadolínio.

\section{Efeitos adversos dos contrastes iodados:}

Foram selecionados 13 artigos que reportaram os efeitos adversos do uso de contraste iodado em diferentes amostras. A tabela 1 mostra a prevalência de efeitos adversos encontrados nos artigos consultados, indicando que a prevalência geral foi baixa na maioria dos trabalhos. Os contrastes iodados, apesar de melhorar a visualização das estruturas anatômicas, podem provocar reações adversas indesejáveis, sendo geralmente classificadas, quanto à etiologia, em reações do tipo anafilactoide e reações quimiotóxicas. As reações anafilactoides, ou idiossincráticas, não dependem da dose de contraste administrada e assemelham-se às reações alérgicas, manifestando-se como urticária, coriza nasal, hipotensão, broncoespasmo, edema laríngeo, podendo ainda levar ao choque e insuficiência respiratória severa. Por outro lado, as reações quimiotóxicas, ou não idiossincráticas, são dependentes da dose recebida, e relacionam-se com as características físico-químicas do contraste, como a osmolaridade e ionicidade. Os sinais e sintomas podem incluir sensação de calor, náuseas e vômitos, arritmia cardíaca, hipertensão, insuficiência renal e convulsões. ${ }^{11}$ As reações anafilactoides são consideradas mais graves, pelo fato de serem imprevisíveis e ocorrerem independente da dose administrada, pois não existe profilaxia. Esse tipo não é considerado reação de hipersensibilidade verdadeira, pois não envolve a produção de anticorpos do tipo Imunoglobulinas E ( $\lg E)$, nem é necessária a sensibilização prévia, sendo de origem desconhecida. O mecanismo destas reações pode envolver a produção e/ou liberação de histamina, serotonina, prostaglandinas, bradicinina, leucotrienos, dentre outros sistemas enzimáticos. ${ }^{12,13}$

O colégio Americano de Radiologia (ACR- American College of Radiology) divide as reações anafilactoides em três graus de severidade: (1) Leves: sendo auto limitadas, incluindo náusea, vômito, urticária, prurido, edema cutâneo, coceira, congestão nasal, tonturas, sudorese, calafrios, espirros, conjuntivite e rinorreia; (2) Moderadas: são mais pronunciadas e requer tratamento farmacológico, incluindo urticária difusa, prurido e eritema, hipertensão ou hipotensão leve, bradicardia ou taquicardia, broncoespasmo sem ou com hipóxia, edema de laringe leve; (3) Graves: requerem tratamento hospitalar, incluindo edema facial ou difuso, eritema difuso e hipotensão grave, edema grave de laringe, broncoespasmo, arritmia grave e parada cardiorrespiratória. As reações ainda podem ser do tipo agudas, ocorrendo 30 minutos após a administração do contraste e tardias, quando aparecem após 30 minutos até sete dias após a administração. ${ }^{14}$ Os contrastes iodados podem ser classificados conforme a sua estrutura em iônicos e não-iônicos. O contraste iodado do tipo iônico possui alta osmolalidade variando de 600 a $2100 \mathrm{mOsm} / \mathrm{kg}$, cerca de duas a sete vezes maior que a osmolalidade do plasma $(290 \mathrm{mOsm} / \mathrm{kg})^{7} \mathrm{O}$ contraste do tipo não iônico tem aproximadamente metade da osmolalidade dos contrastes iônicos em solução. ${ }^{7,15}$

Classicamente a forma iônica está associada a um risco maior de reações adversas e, desta forma, o ACR recomenda que pacientes com risco de desenvolvimento de reações adversas utilizem o contraste não iônico. ${ }^{14}$ $\mathrm{O}$ estudo de Li et al., ${ }^{16}$ avaliou 109.255 pacientes chineses que receberam contraste do tipo não iônico para

Tabela 1 - Prevalência geral dos efeitos adversos ao uso de contrastes usados em tomografia computadorizada (iônico ou não-iônico).

\begin{tabular}{|c|c|c|c|c|}
\hline AUTOR & ANO & PERIÓDICO & $\begin{array}{c}\text { NÚMERO } \\
\text { AMOSTRAL* }\end{array}$ & $\begin{array}{l}\text { PREVAIENCIA GERAL DE } \\
\text { EFEITOS ADVERSOS }\end{array}$ \\
\hline Prieto-Garcia A et al. & 2013 & J Investig Allerg Clin Immunol & 106 & Variável conforme o tipo de contraste \\
\hline Pradubpongsa $\mathrm{P}$ et al . & 2013 & Asian Pac J Allergy Immunol & 55.286 & $1,05 \%$ \\
\hline Jung $\mathrm{K}$ et al. & 2012 & Ann Dermatol & 47.338 & $0,12 \%$ \\
\hline Ho J et al. & 2012 & Asian Pac Allergy & 29.962 & $0,16 \%$ \\
\hline Li X et al. & 2015 & Br J Radiol & 109.255 & $0,34 \%$ \\
\hline Gharekhanloo F e Torabian S & 2012 & Iranian J Radiol & 120 & Variável conforme o tipo de contraste \\
\hline Seong J-M et al. & 2013 & J Korean Med Sci & 48.261 & $13,50 \%$ \\
\hline Ahn $\mathrm{Y}-\mathrm{H}$ et al. & 2015 & J Korean Med Sci & 23 & Variável conforme tipo de contraste \\
\hline Callahan $\mathrm{M}$ et al. & 2009 & Radiology & 12. 494 & $0,46 \%$ \\
\hline Davenport $\mathrm{M}$ et al. & 2009 & Radiology & 140. 753 & $0,70 \%$ \\
\hline Zhang B et al. & 2016 & Medicine & 137.473 & $0,31 \%$ \\
\hline Juchem, BC e Dall'Agnol CM & 2007 & Rev.Latina de Enfermagem & 351 & Variável conforme o tipo de contraste \\
\hline Dillman $\mathrm{J}$ et al. & 2007 & AJR & 11.306 & $0,18 \%$ \\
\hline
\end{tabular}

*pacientes ou aplicações do contraste 
exames de TC, verificando uma prevalência de reações adversas de $0.34 \%$, sendo a maioria classificada como reações leve. Estudos que avaliaram os efeitos adversos em relação aos contrastes de baixa osmolaridade e os iso-osmolares verificaram que as reações adversas imediatas foram mais comuns no grupo que recebeu o contraste monomérico de baixa osmolaridade (iopromide) se comparado ao grupo que recebeu o contraste iso-osmolar (iodixanol). As reações encontradas foram principalmente cutâneas (incluindo coceira/prurido e edema)..$^{17-19}$ Outro estudo demonstrou que o contraste mais utilizado em exames de imagem é o iopromide $(45,5 \%)$, e o mesmo teve maior prevalência $(54,2 \%)$ de efeitos adversos se comparado aos demais, bem como em relação ao contraste iso-osmolar (iodixanol), cuja prevalência foi de apenas 7,6\%. Todavia, o mesmo estudo demonstrou uma elevada prevalência de efeitos adversos no trato urinário de pacientes que utilizaram o iodixanol e desta forma, mesmo que o mesmo seja mais recomendado em algumas situações, ele pode ter um componente nefrotóxico a ser elucidado. ${ }^{20}$

Os mecanismos envolvidos nas reações adversas ao uso de contrastes iodados não foram elucidados. Entretanto, pode ser possível a associação entre os eventos adversos e a dose, via de administração e taxa de administração do contraste. ${ }^{21}$ Com relação ao tipo de reações adversas encontradas verificou-se que a maioria dos estudos relatou reações leves do tipo cutâneas. A tabela 2 mostra a relação dos estudos com a prevalência e tipos de manifestações encontradas.

O estudo de Pradubpongsa et al., ${ }^{22}$ avaliou a prevalência de efeitos adversos de doses de contraste aplicadas 55.286 vezes durante três anos, onde em 579 aplicações resultaram em efeitos adversos, destas $95,9 \%$ foram em adultos e 4,1\% em pacientes pediá- tricos (idade inferior a 18 anos). A maioria das reações ocorreu com o uso de contraste não iônico. Entre os pacientes que tiveram reações, $75,8 \%$ possuíam pelo menos uma comorbidade ou doença alérgica. $O$ efeito adverso mais frequente foi cutâneo, respondendo por $75 \%$ das reações imediatas e por $100 \%$ das reações tardias. Dillman et al., ${ }^{23}$ avaliaram os efeitos adversos de meios de contraste não iônico hiposmolar em pacientes menores que 19 anos. Das 11.306 doses administradas, foram reportadas reações adversas em apenas 20 pacientes, sendo a maioria leves.

Alguns estudos demonstraram uma baixa prevalência de reações adversas. ${ }^{24,25}$ Davemport et al. ${ }^{26}$ verificaram a prevalência de reações adversas em 140.753 aplicações intravenosas de contrastes hiposmolares. Eles verificaram uma prevalência de $0,7 \%$ de reações adversas, sendo a maioria leve. Destes efeitos, $18 \%$ ocorrem mesmo com uso de medicação prévia (preparo antialérgico) chamadas de breakthrough reactions, onde $80 \%$ foram classificadas como leve, 18,4\% como moderadas e apenas $1,57 \%$ como severas. As reações leves descritas foram: eritema, congestão nasal, tosse, espirros, prurido, congestão nasal. As reações moderadas incluíram: bradicardia, broncoespasmo, dor no peito, dispneia, edema facial, hipertensão, hipotensão transitória, hipoxemia leve, taquicardia, urticária difusa. As reações severas encontradas foram: parada cardiorrespiratória, hipotensão refratária, hipoxemia moderada ou grave e edema de laringe. ${ }^{26}$ Um estudo recente avaliou 137.473 pacientes que realizaram exames de TC com contrastes do tipo não iônico e hiposmolares. Dentre estes pacientes, $61 \%$ eram do sexo masculino e $0,31 \%$ tiveram reações adversas, sendo a maioria classificadas como leve e com sintomas cutâneos. ${ }^{27} \mathrm{Cabe}$ salientar que dos 13 trabalhos selecionados, apenas

Tabela 2 - Tipos de reações adversas ao uso do contraste iodado.

\begin{tabular}{|c|c|c|c|}
\hline AUTOR & ANO & $\begin{array}{l}\text { PREVAIÊNCIA E CLASSIFICAC̣ÃO } \\
\text { DAS REAC̣ÕES (ACR) }\end{array}$ & REAC̣ÕES MAIS COMUNS \\
\hline Prieto-Garcia A et al. & 2013 & $\begin{array}{c}\text { Leve- } 54,5 \% \\
\text { Moderada- } 36,4 \% \\
\text { Severa- } 9,1 \%\end{array}$ & Cutâneas (urticária e angioedema) \\
\hline Pradubpongsa $\mathrm{P}$ et al. & 2013 & $\begin{array}{l}\text { Leve- } 65,3 \% \\
\text { Moderada- } 32,2 \% \\
\text { Severa- } 2,5 \%\end{array}$ & $\begin{array}{c}\text { Cutâneas (urticária, eritema e angioedema) } \\
\text { Gastrintestinais (náuseas e vômitos) }\end{array}$ \\
\hline Jung $\mathrm{K}$ et al. & 2012 & $\begin{array}{l}\text { Leve e moderada- } 74,7 \% \\
\text { Severa- } 25,3 \%\end{array}$ & Cutâneas (urticária, eritema e angioedema) \\
\hline Ho J et al. & 2012 & $\begin{array}{c}\text { Leve- } 70 \% \\
\text { Moderada- } 28 \% \\
\text { Severa- } 2 \%\end{array}$ & $\begin{array}{c}\text { Cutâneas (urticária, eritema e angioedema) } \\
\text { Respiratória (dispneia) }\end{array}$ \\
\hline Li $X$ et al. & 2015 & $\begin{array}{c}\text { Leve- } 77 \% \\
\text { Moderada- } 19,2 \% \\
\text { Severa- } 3,8 \%\end{array}$ & $\begin{array}{l}\text { Cutâneas } \\
\text { Respiratórias }\end{array}$ \\
\hline Seong J-M et al. & 2013 & Severa- 5,7\% & Cutâneas Respiratórias Gastrintestinais \\
\hline Callahan $\mathrm{M}$ et al. & 2009 & $\begin{array}{l}\text { Leve- } 82 \% \\
\text { Moderada- } 18 \%\end{array}$ & - \\
\hline Davenport $\mathrm{M}$ et al. & 2009 & $\begin{array}{l}\text { Leve- } 93,04 \% \\
\text { Moderada- } 9,48 \% \\
\text { Severa- } 1,6 \%\end{array}$ & $\begin{array}{l}\text { Cutâneas (Eritema, prurido) } \\
\text { Respiratórias (Congestão nasal, broncoespasmo) }\end{array}$ \\
\hline Zhang B et al. & 2016 & $\begin{array}{l}\text { Leve- } 77,1 \% \\
\text { Moderada- } 19,1 \% \\
\text { Severa- } 3,8 \%\end{array}$ & $\begin{array}{l}\text { Cutâneas } \\
\text { Gastrintestinais }\end{array}$ \\
\hline Dillman $\mathrm{J}$ et al. & 2007 & $\begin{array}{l}\text { Leve- } 80 \% \\
\text { Moderada- } 5 \% \\
\text { Severa- } 15 \%\end{array}$ & $\begin{array}{l}\text { Cutâneas (urticária, rash) } \\
\text { Respiratórias (rinorréia) }\end{array}$ \\
\hline
\end{tabular}


um foi realizado no Brasil. ${ }^{28}$ indicando a precariedade de estudos que avaliem a prevalência e magnitude dos efeitos adversos com o uso de contraste iodado no país.

Efeitos adversos dos contrastes usados em Ressonância Magnética

A RM usa contrastes à base de quelantes do íon paramagnético gadolínio $(\mathrm{Gd})$. Estes podem ser divididos em duas categorias, extracelular inespecífico e intracelular específico, sendo que a principal diferença está na molécula quelante que carrega o Gd. ${ }^{10}$ Assim como já foi descrito para os contrastes iodados, o uso de contrastes paramagnéticos também pode desencadear reações anafilactoides ou reações idiossincráticas, caracterizadas por manifestações cardiovasculares, respiratórias ou cutâneas, podendo, em alguns casos, ocasionar reações graves, incluindo choque. As reações adversas descritas incluem: náuseas, cefaleia, lombalgia, vertigens, vasodilatação, alteração do paladar e dor no local da aplicação. ${ }^{29} \mathrm{O}$ presente estudo coletou dados de oito trabalhos publicados nos últimos 10 anos no PudMed. Destes, dois são estudos de caso e seis são artigos originais. A Tabela 3 mostra a taxa de prevalência dos efeitos adversos em relação ao uso dos contrastes usados em RM nos artigos originais.

Dos artigos analisados, dois reportaram reações alérgicas graves ao uso de $\mathrm{Gd}$, sendo desta forma incluídos na presente pesquisa, pois reportaram reações anafilactoides severas envolvendo o trato respiratório e cardiovascular, mas sem óbito. ${ }^{30,31} \mathrm{O}$ estudo de Bruder et al., ${ }^{32}$ incluiu 37.788 doses de contraste a base de gadolínio administrados nos pacientes. O estudo verificou a ocorrência de $0,12 \%$ reações que ocorreram durante ou imediatamente após o procedimento, sendo que $38 \%$ das reações ocorreram em mulheres. O estudo também não revelou diferença entre as reações agudas e a dose administrada, sendo que a maioria das reações adversas foi classificada como leve, incluindo manifestações cutâneas como eritema e rash cutâneo. O estudo de Ishiguchi \&Takahashi, ${ }^{33}$ avaliou os efeitos do gadoterato de meglumina (Gd-DOTA, Magnescope/Dotarem) que é um quelado iônico do gadolínio aprovado para imagens de lesões cerebrais e espinais, dentre outros. O estudo revelou uma prevalência de 0,93\% de reações adversas, sendo a maioria em mulheres $(50,5 \%)$ e classificadas como leves, incluindo alterações cutâneas e gastrintestinais. Outro estudo verificou a prevalência de $0,1 \%$ de efeitos adversos em pacientes submetidos à RM com uma média de idade de 45,7 anos. A taxa de reações adversas foi maior em mulheres se comparado aos homens, sendo a maioria das reações classificadas como leves. ${ }^{34}$ Em contrapartida, um estudo que avaliou 105.607 injeções de contrastes a base de gadolínio não encontrou associação entre o sexo feminino e a ocorrência de reações alérgicas, sendo que em ambos os sexos, a maioria das reações foram classificadas como leves. ${ }^{35} \mathrm{~A}$ tabela 4 mostra os principais efeitos adversos com o uso do Gd.

Tabela 3 - Prevalência geral dos efeitos adversos ao uso de contrastes usados em RM.

\begin{tabular}{|c|c|c|c|c|}
\hline AUTOR & ANO & PERIÓDICO & $\begin{array}{c}\text { NÚMERO } \\
\text { AMOSTRAL* }\end{array}$ & $\begin{array}{l}\text { PREVAIÊNCIA GERAL DE } \\
\text { EFEITOS ADVERSOS }\end{array}$ \\
\hline Bruder $\mathrm{O}$ et al. & 2015 & $\mathrm{~J}$ of Cardiovascular Magnetic Ressonance & 37.788 & $0,12 \%$ \\
\hline Ishiguchi \& Takahashi & 2010 & Drugs & 3.444 & $0,93 \%$ \\
\hline Aran $\mathrm{S}$ et al. & 2015 & Clinical Radiology & 194.400 & $\begin{array}{l}\text { Variável conforme o tipo de contraste } \\
\text { (variou de 0,09 a 0,88\%) }\end{array}$ \\
\hline Dillman JR et al. & 2007 & AJR & 78.353 & $\begin{array}{c}0,07 \% \text { em adultos e } 0.044 \% \\
\text { em pacientes pediátricos }\end{array}$ \\
\hline Jingu $A$ et al. & 2014 & BMC Medical Imaging & 20.422 & $0,13 \%$ \\
\hline Davenport MS et al. & 2013 & Radiology & 105. 607 & $0,15 \%$ \\
\hline
\end{tabular}

*pacientes ou aplicações do contraste

Tabela 4 - Tipos de reações adversas relacionadas ao uso de contrastes à base de gadolínio.

\begin{tabular}{|c|c|c|c|}
\hline AUTOR & ANO & $\begin{array}{c}\text { PREVAIÊNCIA E CLASSIFICAC̣ÃO } \\
\text { DAS REAC̣ÕES (ACR) }\end{array}$ & REAC̣ÕES MAIS COMUNS \\
\hline Bruder O et al. & 2015 & $\begin{array}{l}\text { Leve- } 95,5 \% \\
\text { Severa- } 4,5 \%\end{array}$ & $\begin{array}{c}\text { Cutâneas (rubor, eritema) } \\
\text { Gastrintestinais (Náuseas) } \\
\text { Respiratórias (broncoespasmo) }\end{array}$ \\
\hline Ishiguchi \&Takahashi & 2010 & $\begin{array}{l}\text { Leve- } 90 \% \\
\text { Moderada- } 10 \%\end{array}$ & $\begin{array}{c}\text { Cutâneas (erupções, urticária) } \\
\text { Gastrintestinais (Náusea, vômito) } \\
\text { Neurológicas (dor de cabeça, gosto ruim estranho) }\end{array}$ \\
\hline Aran S et al. & 2015 & $\begin{array}{l}\text { Leve }-83,3 \% \\
\text { Moderada- } 13,7 \% \\
\text { Severa- } 2,4 \%\end{array}$ & $\begin{array}{l}\text { Gastrintestinais (Náusea) } \\
\text { Cutâneas (rash e urticária) }\end{array}$ \\
\hline Davenport MS et al. & 2013 & $\begin{array}{l}\text { Leve- } 84,5 \% \\
\text { Moderada- } 11,8 \% \\
\text { Severa- } 3,7 \%\end{array}$ & $\begin{array}{l}\text { Cutâneas (urticária, eritema, rash facial) } \\
\text { Respiratórias (espirros, congestão nasal, dispneia, } \\
\text { espasmo de laringe) }\end{array}$ \\
\hline Jingu $A$ et al & 2014 & $\begin{array}{l}\text { Leve- } 66,7 \% \\
\text { Moderada- } 33,3 \%\end{array}$ & - \\
\hline Dillman $\mathrm{J}$ et al. & 2007 & $\begin{array}{l}\text { Leve-74\% } \\
\text { Moderada- } 19 \% \\
\text { Severa- } 7 \%\end{array}$ & $\begin{array}{l}\text { Cutâneas (urticária, rash) } \\
\text { Respiratórias (dificuldade em respirar, dor } \\
\text { torácica, hipóxia) }\end{array}$ \\
\hline
\end{tabular}


Jingu et al., ${ }^{36}$ além de verificarem uma prevalência de $0,13 \%$ de efeitos adversos em pacientes submetidos à $\mathrm{RM}$ com contraste, também verificaram a presença de efeitos contrários em 54 indivíduos que realizaram exames de RM com protocolo de preparo antialérgico. Os motivos para o preparo incluíram histórico de reações adversas ao contraste iodado, histórico de reações agudas ao gadolínio, portador de asma e outros. Dos pacientes que utilizaram o preparo, apenas um teve uma reação leve recebendo a pré-medicação por histórico de asma, sendo que o único sintoma referido foi náusea. Outra pesquisa também verificou, além da prevalência de $0,07 \%$ de reações adversas, a presença de fatores de risco associados aos efeitos adversos do contraste. Cinquenta por cento dos pacientes que tiveram reações alérgicas agudas ao contraste tinham um ou mais fatores de risco (histórico de reações alérgicas prévias ao gadolínio, alergia ao contraste iodado ou asma). ${ }^{37}$

\section{DISCUSSÃO}

O presente estudo verificou a ocorrência de reações adversas ao uso de meios de contrastes utilizados em exames de RM e TC. As reações adversas agudas ao meio de contraste ocorrem em uma determinada taxa, conforme o tipo de contraste. No caso de contrastes iodados verificou-se uma taxa de reações adversas de cerca de 0,1 a $13 \%$, enquanto que para os contrastes a base de gadolínio a taxa variou de 0,07 a 0,9\%, sendo menor que para os contrastes iodados. O início das reações adversas aos contrastes é geralmente rápido, ou seja, nos iodados, cerca de $70 \%$ das reações ocorre dentro de 5 minutos após a injeção, e $96 \%$ das reações de graves manifestam-se dentro de 20 minutos. ${ }^{38}$ Geralmente, as reações são leves e incluem: prurido e urticária/angioedema seguidos de náuseas e vômitos. Em alguns casos também ocorrem reações mais graves envolvendo o sistema respiratório e cardiovascular. As reações tardias ocorreram em horas a vários dias após o uso do contraste incluindo erupção medicamentosa fixa, eritema exsudativo multiforme, eosinofilia relacionada com a droga. Geralmente são reações classificadas como leves ou moderadas.

Outro achado do presente estudo foi a verificação de que a maioria dos efeitos colaterais dos contrastes iodados foi associado aos contrastes de baixa osmolaridade em comparação com os contrastes iso-osmolares. ${ }^{20,26}$ A maioria das reações adversas aos meios de contraste iodados é leve, tais como náuseas, vômitos, urticária e prurido, contudo, podem ocorrer efeitos mais graves, incluindo hipotensão, parada respiratória e cardíaca e convulsões. A incidência destes eventos adversos diminuiu consideravelmente com o uso de meios de contraste de baixa osmolaridade. Embora a incidência global de efeitos adversos tenha diminuído com o uso destes contrastes, os eventos continuam a ocorrer, e, desta forma, não é claro se existem diferenças entre os diversos tipos de contrastes hiposmolares. Reação adversa aos meios de contraste iodados pode envolver a liberação de histamina e outros mediadores inflamatórios de mastócitos e basófilos. Possivelmente ocorre devido a um efeito direto sobre as células ou indireto através da ativação do sistema complemento. No entanto, resultados positivos em testes cutâneos, testes de ativação de basófilos e específicos para a detecção de $\lg$ E têm sido cada vez mais relatados. ${ }^{38}$

A maioria dos eventos adversos relacionados ao gadolínio é considerada leve e geralmente associada a fatores como: histórico de reação anterior ao contraste, sexo feminino, presença de asma e outras alergias. Ainda não está claro se as taxas de reação dependem da estrutura dos diferentes meios de contraste à base de gadolínio. As manifestações clínicas aos meios de contraste usados em RM não são diferentes das reações associadas ao contraste iodado, sendo que os sintomas mais comuns são: urticária e náuseas, enquanto que reações severas são raras. De maneira similar ao contraste iodado, os mecanismos envolvidos na patogênese das reações alérgicas associadas ao gadolínio não estão esclarecidos. O uso de pré-medicação, ou seja, o preparo antialérgico (geralmente feito com corticosteroides e anti-histamínicos) também é discutível. Embora estudos tenham demonstrado a importância do preparo, o seu uso não é consensual em relação ao custo benefício para o paciente e a sua efetividade na redução de efeitos adversos. ${ }^{35,36}$

A presente pesquisa avaliou a ocorrência das reações de hipersensibilidade relacionadas ao uso de contraste. $\mathrm{O}$ mecanismo de ação destas reações pode estar associado à quantidade de cátions liberada pelo contraste, expansão do volume plasmático, vasodilatação generalizada por efeito na musculatura lisa e lesão do endotélio vascular. Destaca-se ainda a ocorrência de reações combinadas ou complexas e, desta forma, recomenda-se seguir as indicações do manual do ACR em relação aos meios de contraste, que sugere o uso de preparo antialérgico para pacientes com risco de reações adversas. Recomenda-se também, a realização do exame em hospitais/clínicas que tenham estrutura para o manejo dos efeitos adversos que podem ocorrer, além da permanência do paciente no serviço por cerca de 30 minutos após a realização do exame, tendo em vista que algumas reações moderadas podem ocorrer mais tardiamente.

\section{CONSIDERACÕES FINAIS}

Através da análise feita no presente trabalho, observa-se que a prevalência de eventos adversos relacionados aos contrastes iodados é maior do que os associados aos contrastes baseados no gadolínio, fato que destaca a importância da pesquisa, pois mundialmente são realizadas mais tomografias do que ressonâncias principalmente pela diferença de custo entre os exames. Contudo, a maioria das reações observadas é classificada como leve a moderada e somente poucas consideradas graves. Ainda, os mecanismos envolvidos no desenvolvimento destes efeitos permanecem desconhecidos, fato que instiga o desenvolvimento de pesquisas nesta área. Por fim, destaca-se a importância da avaliação prévia do paciente a ser submetido ao exame quanto ao seu histórico de reações adversas ou alergias, tendo em vista que a escolha do meio de contraste a ser utilizado deve ser feita, visando minimizar os efeitos adversos que podem ser induzidos. 


\section{REFERÊNCIAS}

1. Carvalho, ACP. História da tomografia computadorizada. Revista Imagem 2007;29(2):61-66.

2. Amorim, AS, Pinto Júnior, VL, Shimizu, HE. O desafio da gestão de equipamentos médico-hospitalares no Sistema Único de Saúde. Saúde Debate 2015;39(105):350-362. doi: 10.1590/0103-110420151050002004

3. Martins, L. O Segmento da medicina diagnostica no Brasil. Revista Faculdade Ciências Médicas 2014;16(3):139 - 145.

4. Freitas, MB, Yoshimura, EM. Levantamento da distribuição de equipamentos de diagnóstico por imagem e da freqüência de exames radiológicos no estado de São Paulo. Radiologia Brasileira 2005;38(5):347--354. doi: 10.1590/S010039842005000500008

5. Rogers LF. "My word, what is that?": Hounsfield and the triumph of clinical research. American Journal Roentgenology 2003;180:1501-1501. doi:10.2214/ajr.180.6.1801501.

6. Stimac GK, Melgaço ALS. Introdução Ao Diagnóstico Por Imagens. Rio De Janeiro: Guanabara Koogan, 1992.467p.

7. Thomsen HS, Morcos SK. Radiographic Contrast Media. BJU Intl 2000;86(1):1-10.

8. Santos AP, Gaivão AM, Tavares A, Ferreira S. Produtos de contrastes iodados. Acta Med Port 2009; 22: 261-274.

9. Ba-Ssalamah A, Uffmann M, Saini S, Bastati N, Herold C, Schima W. Clinical Value Of MRI Liver-Specific Contrast Agents: A Tailored Examination For A Confident NonInvasive Diagnosis Of Focal Liver Lesions. Eur Radiol 2009; 19:342-57. doi: 10.1007/s00330-008-1172-x

10. Elias JR, Santos AC, Koenigkam-Santos M, Nogueira-Barbosa $\mathrm{MH}$, Muglia VF. Complicações Do Uso Intravenoso De Agentes de Contraste À Base De Gadolínio Para Ressonância Magnética. Radiol Bras 2008;4(4):263-267.

11. Silva EA. Meios De Contraste lodado. In: Oliveira Lan, Editor. Assistência À Vida Em Radiologia: Guia Teórico e Prático. São Paulo (SP): Colégio Brasileiro De Radiologia; 2000.p.16-114.

12. Hagan JB: Anaphylactoid And Adverse Reactions To Radiocontrast Agents. Immunol Allergy Clin N Am 2004; 24:507-519. doi:10.1016/j.iac.2004.03.005

13. Maddox TG. Adverse Reactions to Contrast Material: Recognition, Prevention, And Treatment. Am Fam Physician 2002;66(7):1229-34.

14. American College of Radiology (ACR) Committee on Drugs and Contrast Media. ACR manual on contrast media, version 9. Acesso em 28 de maio de 2016. Disponível em: https:// www.acr.org/Quality-Safety/Resources/Contrast-Manual

15. Christiansen C: X-ray Contrast Media - An overview. Toxicol 2005;209:185-7. doi:10.1016/j.tox.2004.12.020

16. Li X, Chen J, Zhang L, Liu H, Wang S, Chen X, Fang J, Wang S, Zhang W. Clinical observation of the adverse drug reactions caused by non-ionic iodinated contrast media: results from 109,255cases who underwent enhanced CT examination in Chongqing, Br J Radiol 2015;88:20140-491. doi: 10.1259/bjr.20140491

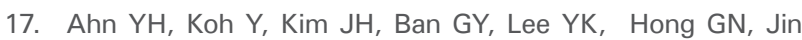
UR,Choi BJ,Shin YS,Park HS,YeYM. The Potential Utility of lodinated Contrast Media (ICM) Skin Testing in Patients with ICM Hypersensitivity J Korean Med Sci 2015;30:245-251 doi:10.3346/jkms.2015.30.3.245

18. Prieto-Garcia A, Tomás M, Pineda R, Tornero P, Herrero T, Fuentes V, Zapatero L, de Barrio M. Skin Test-Positive Immediate Hypersensivity reaction to iodinate contrast media: The role of controlled challenge testing. J Investig Allerg Clin Immunol 2013;23(3):183-189.
19. Gharekhanloo F, Torabian S. Comparison of Allergic Adverse Effects and Contrast Enhancement Between lodixanol and lopromide. Iran J Radiol 2012;9(2):63-66. doi:10.5812/ iranjradiol.7696.

20. Seong JM, Choi NK, Lee J, Chang Y, Kim YJ, Yang BR, Jin, XM, Kim JY, Park BJ. Comparison of the Safety of Seven lodinated Contrast Media. J Korean Med Sci 2013;28:17031710. doi:10.3346/jkms.2013.28.12.1703

21. Federle MP, Willis LL, Swanson DP. Ionic versus nonionic contrast media: a prospective study of the effect of rapid bolus injection on nausea and anaphylactoid reactions. J Comput Assist Tomogr 1998;22(3):341-345.

22. Pradubpongsa $P$, Dhana, N, Jongjarearnprasert K, Janpanich $S$ and Thongngarm $T$ Adverse reactions to iodinated contrast media: prevalence, risk factors and outcome - the results of a 3-year period Asian Pac J Allergy Immunol 2013. 31:299306.doi: 10.12932/AP0297.31.4.2013

23. Dillman J, Strouse PJ, Ellis JH, Cohan RH , Jan SC. Incidence and Severity of AcuteAllergic-Like Reactions to IV Nonionic lodinated Contrast Material in Children. AJR 2007;188; 1643-47.

24. Ho J, Kingston RJ, Young NY, Katelaris $\mathrm{CH}$, and Sindhusake D, Immediate hypersensitivity reactions to IV nonionic iodinated contrast in computed tomography. Asia Pac Allergy 2012;2:242-247. doi:10.5415/apallergy.2012.2.4.242

25. Jung KE, Chung J, Park BC, Jee KN, Jee YK, Kim MH. A Clinical Study of Cutaneous Adverse Reactions to Nonionic Contrast Media in Korea. Ann Dermatol 2012;24(1):22-25. doi: 10.5021/ad.2012.24.1.22

26. Davenport MS, Cohan RH, Caoili, EM, Ellis JH. Repeat Contrast Medium Reactions in Premedicated Patients: Frequency and Severity. Radiology 2009;253:(2):372-379. doi: 10.1148/radiol.2532090465

27. Zhang $B$, Dong $Y$, Liang, L , Lian, Z , Liu J, Luo X, Chen W, Li X, Liang C, Zhang S. The Incidence, Classification, and Management of Acute Adverse Reactions to the Low-Osmolar lodinated Contrast Media Isovue and Ultravist in ContrastEnhanced Computed Tomography Scanning. Medicine 2016; 95(12):1-9. doi: 10.1097/MD.0000000000003170

28. Juchem BC, Dall'Agnol CM. Reações adversas imediatas ao contraste iodado intravenoso em tomografia computadorizada Rev Latino-am Enfermagem 2007;15(1).

29. Van Beers BE, Pastor CM, Hussain HK. Primovist, Eovist: What To Expect? J Hepatol 2012;57:421-9. doi:10.1016/j. jhep.2012.01.031

30. Singer BD, Oodrick RS, Pedicano JB: Severe Adverse Drug Reaction to Gadobenate Dimeglumine The Scientific World journal 2009;9:363-365. doi 10.1100/tsw.2009.41

31. Park J, Byun IIH, Park KH, Lee J-H, Nam EJ, Park J-W. Acute Respiratory Distress Syndrome after the Use of Gadolinium Contrast Media Yonsei Med J 2015;56(4):1155-1157.doi: 10.3349/ymj.2015.56.4.1155

32. Bruder O, Schneider S, Pilz G, van Rossum AC, Schwitter J, Nothnagel D, et al. Update on Acute Adverse Reactions to Gadolinium based Contrast Agents in Cardiovascular MR. Large Multi-National and Multi-Ethnical Population Experience With 37788 Patients From the EuroCMR Registry Journal of Cardiovascular Magnetic Resonance 2015;17(58):2-7. doi: 10.1186/s12968-015-0168-3.

33. Ishiguchi T and Takahashi S. Safety of Gadoterate Meglumine (Gd-DOTA) as a Contrast Agent for Magnetic Resonance Imaging Results of a Post-Marketing Surveillance Study in Japan. Drugs 2010;10(3):133-145. doi: 10.2165/11539140- 


\section{0-00000.}

34. Aran S, Shaqdan KW, Abujudeh HH. Adverse allergic reactions to linear ionic gadolinium-based contrast agents: experience with 194, 400 injections Clinical Radiology 2015;70:466-475. doi: 10.1016/j.crad.2014.12.011

35. Davenport MS, Dillman JR, Hussain CHK, Khalatbari $\mathrm{S}$, McHugh, JB, Ellis JH. Effect of Abrupt Substitution of Gadobenate dimeglumine for Gadopentetate Dimeglumine on Rate of Allergic-like Reactions. Radiology 2013;266 (3):773-782.

36. Jingu A, Fukuda J, Taketomi-Takahashi A, Tsushima Y. Breakthrough reactions of iodinated and gadolinium contrast media after oral steroid premedication protocol BMC Medical Imaging 2014;14(34):1-6. doi: 10.1186/1471-2342-14-34

37. Dillman JR, Ellis JH, Cohan RH, Strouse PJ, San SC, Frequency and Severity of Acute Allergic-Like Reactions to Gadolinium-Containing IV Contrast Media in Children and Adults AJR 2007;189:1533-1538.

38. Brockow K, Romano A, Aberer W, Bircher AJ, Barbaud A, Bonadonna $\mathrm{P}$ et al. European Network of Drug Allergy and the $\mathrm{EAACl}$ interest group on drug hypersensitivity. Skin testing in patients with hypersensitivity reactions to iodinated contrast media - a European multicenter study. Allergy. 2009;64:234-41. doi: 10.1111/j.1398-9995.2008.01832.x.

Como citar: POZZOBON, Adriane; TRINDADE, Fernanda Rocha da. Avaliação das reações adversas ao uso de contrastes em exames de diagnóstico por imagem. Cinergis, Santa Cruz do Sul, v. 18, n. 4, out. 2017. ISSN 2177-4005. Disponível em: <https:// online.unisc.br/seer/index.php/cinergis/article/view/10919>. Acesso em: 07 dez. 2017. doi:http://dx.doi.org/10.17058/cinergis. v18i4.10919. 